Ann. Abeille, I962, 5 (2), I35-I43.

\title{
LA SÉLECTION CONVERGENTE, MÉTHODE PRATIQUE POUR L'AUGMENTATION DE L'EFFICACITÉ DE LA POLLINISATION PAR LES INSECTES
}

\author{
E. V. SANDULEAC \\ Station centrale de Recherches pour la Sériciculture et l'Apiculture, \\ Bucarest-Baneasa (Roumanie).
}

\section{SOMMAIRE,}

On entend par sélection convergente une action simultanée sur la plante en vue d'auginenter son pouvoir attractif sur la faune pollinisatrice et une action sur l'Insecte en vue d'améliorer ses qualités de pollinisateurs.

Étudiant plus spécialement la sécrétion nectarifère des plantes cultivées, l'auteur montre que de grandes différences existent sous ce rapport d'une variété à l'autre. Il préconise la sélection des variétés cultivées en tenant compte de leur valeur mellifère.

\section{INTRODUCTION}

Les plantes entomophiles et l'entomofaune pollinisatrice constituent une unité dont l'évolution phylogénétiques a le même sens. L a sélection naturelle a déterminé au cours des âges, la survie des espèces végétales qui, une fcis adaptées à la pollinisation entomophile, ont le plus intéressé les Insectes pollinisateurs parce qu'elles fournissaient le nectar et le pollen indispensables à leur existence. Les progrès de l'agriculture et le souci de l'homme de développer au maxiumum les caractères utiles des plantes cultivées, ont amené une certaine rupture de l'équilibre biologique. Ce fut DARWIN qui attira le premier l'attention sur ces faits dans les œuvres célèbres que sont "L'origine des espèces " (I859) et "Les effets de la pollinisation croisée et de l'autofécondation $)(\mathrm{I} 876)$.

Les idées de DARWIN ont été reprises d'une manière particulièrement intéressante par Ivan KLINGEN (I85I-I922), créateur d'un système d'agriculture utilisant les Abeilles. I1 a développé 1'idée de la sélection convergente (en russe "Vstretnaia Selectia») 
dont le but est le perfectionnement de la symbiose qui existe naturellement entre les Abeilles et certaines plantes. Le travail porte simultanément sur la plante afin de sélectionner les formes les mieux adaptées à la pollinisation par les Abeilles et aussi sur l'Abeille elle-même pour amplifier ses qualités de pollinisatrice.

L'idée de la sélection convergente a été souvent l'objet de discussions contradictoires. Des hommes de science aussi connus que I. M. KLINGEN, J. Martinet, E. IINDHARD, U. ZoFkA, P. I. Lisitin, A. F. Gubin, etc. ont précisé que le but principal de la sélection convergente est la réalisation d'un rapprochement morpho-physiologique entre 1'Abeille et les plantes dont elle tire sa subsistance.

L'application de la sélection convergente aux végétaux a été marquée par certains succès ; nous mentionnerons ceux qui ont été obtenus sur le Trèfle (N. P. SMARAGDovA, D. M. Savceniuc, P. B. Socolovskaia, etc.,) le Tournesol (A. S. Rozov, etc.), les Arbres fruitiers (A. S. Tatarintev, V. K., Rimasevski, etc.), la Lavande (Berbie, I958), ainsi que les succès obtenus par D. V. BuRCH qui a découvert une corrélation entre l'arôma et la teneur en sucres du nectar de Tilia, Nicotina affinis, etc., ce qui constitue un élément nouveau dans l'étude de la sélection convergente.

L'étude des travaux publiés jusqu'ici sur la sélection convergente nous amène à la conclusion que cette méthode, efficace pour l'amélioration de la pollinisation entomophile, ne doit pas se limiter à un simple rapprochement morphologique entre la plante et l'Abeille, mais doit tendre également vers un objectif physiologique, le développement de la sécrétion du nectar et de tous les organes floraux qui jouent un rôle dans l'attraction qu'exerce la fleur sur l'insecte pollinisateur. Une meilleure compréhension des rapports qui existent entre le végétal et l'insecte nous amènera fatalement à des résultats intéressants. La réalisation des objectifs de la sélection convergente donnera aux sélectionneurs la possiblité de transformer les étendues cultivées de plantes entomophiles en une précieuse réserve mellifère, en même temps qu'elle permettra d'améliorer leur pollinisation.

Pour mettre en évidence les conséquences économiques de notre conception de la sélection convergente, nous montrerons plus loin les résultats d'expériences réalisées sur le Tournesol, le Pommier et le Robinier. Quant au 'Trèfle, nous considérons que les travaux réalisés à ce jour apportent une réponse concluante et qu'ils montrent la voie dans laqualle doit s'engager notre action pour obtenir des résultats susceptibles de vastes applications.

\section{MÉTHODE DE' 'TRAVAIL,}

La détermination de la valeur mellifère, caractéristique essentielle du pouvoir attractif des plantes étudiées, a été faite au moyen de la méthode de prélèvement du nectar par tubes capillaires. On a utilisé des capillaires de formes diverses et on a pesé le nectar extrait avec une balance de torsion. Ia concentration en sucres du nectar a été ensuite déterminée avec un réfractomètre donnant des valeurs de o, à 85 p. ioo. Des données obtenues on déduit par le calcul la production de miel par ha. Ia méthode employée est donc très simple mais tout à fait sulfisante pour mettre en évidence les éléments relatifs ì la sélection convergente sur le plan du végétal.

\section{PRINCIPAUX RÉSULTATS}

Pour le Tournesol, le Pommier et le Robinier, nous avons constaté l'existence d'une corrélation directe entre la production de sucre par les nectaires et l'attractivité de la plante. Ises variétés qui présentent la plus forte production de nectar sont aussi 
celles qui sont très visitées par les Abeilles et donnent une forte production de graines ou de fruits, car leur pollinisation est très complète. Pour le Tournesol, il a été établi qu'il existe une corrélation positive entre la production de nectar et la teneur en huile des graines.

TABI,EAU I

Valeur mellifère du Tournesol.

\begin{tabular}{|c|c|c|c|c|}
\hline Variétés & $\begin{array}{c}\text { Sucre interverti } \\
\text { mg/Heur }\end{array}$ & $\begin{array}{l}\text { Saccarose } \\
\text { mg/fleur }\end{array}$ & $\begin{array}{c}\text { Sucres totaux } \\
\text { morfleur }\end{array}$ & $\begin{array}{l}\text { Niel } \\
\mathrm{kg} / \mathrm{has}\end{array}$ \\
\hline Population locale ........ & 0,166 & 0,100 & 0,266 & 23,940 \\
\hline Vnïmk ............ & 0,192 & 0,192 & 0,321 & $2 \times, 8,80$ \\
\hline Vniimk isio .... & 0,298 & 0,092 & 0,390 & 35,100 \\
\hline Armavir : $: 97 \ldots \ldots \ldots$ & $0,1,50$ & $0,2 \times 6 i$ & 0,736 & $0,2,1 ; 0$ \\
\hline
\end{tabular}

Dans le tableau I nous donnons le résultat de mesures de sécrétion nectarifère qui ont porté sur quatre variétés de 'Tournesol. La production de nectar la plus forte est celle de la variété Armavir 3497 (o,736 mg de sucre par fleur), puis viennent deux variétés $V$ nimk (respectivement 0,390 et $0,32 \mathrm{I} \mathrm{mg}$ ) et enfin un Tournesol appartenant à une plantation locale $(0,266 \mathrm{mg})$. Sous le rapport de la production de miel par ha, on retrouve le même classement. Ces résultats ont été confirmés sur toute l'étendue du pays et dans des conditions de production variées. Le Tournesol provenant de populations locales est peu recherché parles Abeilles et, en conséquence sa production de graines est restée à un niveau toujours bas. Par contre, les variétés Vniimk et Armavir sont recherchées par les Abeilles, de telle sorte que la sélection continue axée sur la production de nectar et d'huile aboutira à la création de variétés de plus en plus intéressantes.

TABLEAU 2

Valeur mellifère du l'ommier.

\begin{tabular}{|c|c|c|c|c|c|c|}
\hline \multirow{2}{*}{ Variétés } & \multicolumn{3}{|c|}{ mg/fleur en 2 it heures } & \multicolumn{3}{|c|}{ Concentration en sucres $\%$} \\
\hline & Min. & Max. & Moyenne & Min. & Max. & Moyenne \\
\hline Cretesc .......... & 0,22 & 3,71 & 1,4 & $1 i, 0$ & 50,6 & $8 i, 9$ \\
\hline Reinette Iaumann.. & 0,07 & 1,88 & 0,77 & 16,0 & 48,8 & $3:, 4$ \\
\hline Reinette llorée.... & 0,15 & 2,09 & 1,9 & 30,6 & 46,3 & 35,7 \\
\hline $\begin{array}{r}\text { Reinette de Cham- } \\
\text { pagne .......... }\end{array}$ & 0,15 & 0,56 & $0,3: 2$ & 12,6 & 32,0 & 21,9 \\
\hline Gustar ............ & 0,20 & 1,35 & 0,60 & 16,0 & $\begin{array}{l}3,0 \\
3:, 0\end{array}$ & 23,2 \\
\hline Astrakan kouge.... & 0,15 & 1,03 & 0,60 & 16,0 & 30,2 & 21,2 \\
\hline Jonathan ......... & 0,13 & 0,99 & 0,58 & 23,8 & 40,0 & 32,2 \\
\hline Golden l'armen & 0,21 & 0,89 & $0,1 x$ & $2 !, 0$ & 28,1 & 25,4 \\
\hline Patul. . . . . . . . & 0,11 & 1,03 & 0,36 & 14,0 & 31,1 & $2 \%, 9$ \\
\hline Masanski ........ & 0,10 & $0,9^{\prime}$ & $0,4^{\prime}{ }^{\prime}$ & $20, t_{t}$ & $\because 0,0$ & 33,9 \\
\hline London Pepring.... & 0,28 & 1,01 & 0,62 & 26,0 & 38,2 & 31,6 \\
\hline Boiken ........... & 0,21 & 2,24 & 0,67 & $3 i, 0$ & 40,2 & 36,2 \\
\hline Signe Tillesh ...... & 0,78 & 2,03 & 1,12 & 19,0 & $3 \mathrm{~h}, \mathrm{~s}$ & 27,5 \\
\hline
\end{tabular}


TABLEAU 2 A

Valeur mellifère de quelques espèces horti-viticoles.

\begin{tabular}{|c|c|c|c|c|c|}
\hline Espèces et variétés & $\begin{array}{l}\text { Caractéristique } \\
\text { de la plante }\left(^{*}\right)\end{array}$ & $\begin{array}{l}\text { Période } \\
\text { de floraison } \\
\text { (mois) }\end{array}$ & $\begin{array}{l}\text { Couleur } \\
\text { des fleurs }\end{array}$ & $\begin{array}{l}\text { Sucre } \\
\text { par fleur } \\
\text { (mg) }\end{array}$ & $\begin{array}{c}\text { Miel } \\
(\mathrm{kg} / \mathrm{ha})\end{array}$ \\
\hline 1 & 2 & 3 & 4 & 5 & 6 \\
\hline Anygdalus communis.... & A $n / p / m$ & III-V & rose & - & 6,0 \\
\hline Armeniaca vulgaris...... & A $n / p / m$ & III-IV & rose-blanc & 0,4 & 15 \\
\hline Castanea sativa ........ & A $n / p / m / c$ & VI-VII & jaune & 0,07 & 30 \\
\hline Cerasus avium .......... & A $n / p / m$ & IV $\cdot V$ & blanc & $0,5-1,4$ & $\begin{array}{l}30 \\
(21-36)\end{array}$ \\
\hline Bistritene .... & - & 一 & - & 0,47 & 28 \\
\hline The earliest..$\ldots \ldots \ldots$ & 一 & - & 一 & 0,58 & $3 / t^{\prime}$ \\
\hline Germersdorf ......... & - & - & 一 & 0,72 & 43 \\
\hline Hedelfinger ......... & - & - & - & 0,74 & $4 \prime$ \\
\hline Early black .......... & - & $\cdots$ & - & $0,85(1,10)$ & $51(66)$ \\
\hline 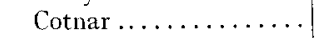 & - & - & - & 0,67 & 40 \\
\hline Dönisen............ & - & - & 一 & $1,10(1,30)$ & $66(78)$ \\
\hline Napoléon ........... & $\longrightarrow$ & - & - & 0,59 & 35 \\
\hline Ramon Oliva......... & - & 一 & 一 & 0,49 & 29 \\
\hline Cerasus vulgaris ....... & A $n / p / m$ & IV-V & $\ldots$ & $0,5 \quad 1,2$ & $30(34)$ \\
\hline Bistritène . . . . . . . & - & - & 一 & $0,54(0,84)$ & $32(50)$ \\
\hline Crisana ............. & 一 & - & 一 & $0,73(1,32)$ & $43(78)$ \\
\hline & & & & 0,50 & 30 \\
\hline Grosser Gobert ....... & - & $\longrightarrow$ & - & 0,66 & 39 \\
\hline Hortensia . . . . . . . & -. & 一 & - & $0,88(1,55)$ & $26(46)$ \\
\hline Maroco Gabor ......... & 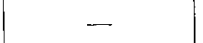 & $\longrightarrow$ & - & $0,45(0,75)$ & $14(32)$ \\
\hline Mocanesti .......... & - & $\longrightarrow$ & - & 0,57 & $17(40)$ \\
\hline 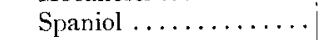 & - & - & - & $0,77(0,96)$ & $23(57)$ \\
\hline Early french ......... & - & - & - & $\begin{array}{l}0,78 \quad 0,81 \\
(1,23)\end{array}$ & $\begin{array}{c}46 \\
(33)\end{array}$ \\
\hline Turkish .... & - & - & blanc & $0,5 / \mathrm{k}$ & 16 \\
\hline Coryllus sp...... & $A p / m$ & III jaune & verdâtre & $\stackrel{(1,06)}{-}$ & $\begin{array}{r}(22) \\
(5)\end{array}$ \\
\hline Cornus mas ..... & A $n / p / c$ & III-IV & jaune & - & $(10)$ \\
\hline Cydonia sp....... & $\Lambda n / p / m$ & IV-V & blanc & 3,4 & $5(3-6)$ \\
\hline Fragaria sp. .......... & $p r n / p$ & IV-V (X) & blanc & $3,4_{1}$ & (5) \\
\hline Grossularia $s p . \ldots \ldots \ldots$ & $a n / p / m$ & IV $\cdot V$ & rougeâtre vert & $1,0-1,10$ & 25 \\
\hline Giant $\ldots \ldots \ldots \ldots \ldots$ & - & - & - & $0,95-1,25$ & 25 \\
\hline Local .............. & 一 & - & $\ldots$ & $0,70-1,10$ & 20 \\
\hline Juglans sp. .......... & A $p / n$ & IV $-\mathrm{V}$ & verdâtre & 一 & $(m: 5)$ \\
\hline Malus communis si sp... & A $n / p / m$ & IV-V & blanc & $0,7(1,6-2,3)$ & $30(34)$ \\
\hline Astrakan rouge ....... & - & - & - & 0,60 & 25 \\
\hline Boiken............. & 一 & - & 一 & 0,85 & 36 \\
\hline Cretesc $\ldots \ldots \ldots \ldots$ & - & 一 & - & $0,60(1,44)$ & $25(61)$ \\
\hline Gustar ........... & - & - & - & 0,78 & 33 \\
\hline Jonathan ........... & 一 & 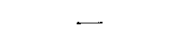 & 一 & $0,58(1,6)$ & $25(28)$ \\
\hline London Pepping ...... & -. & - & - & $0,90(2,04)$ & $38,5(38)$ \\
\hline Masanschi ............ & - & - & -- & 0,55 & 28 \\
\hline Golden Parmen ....... & - & - & $\longrightarrow$ & $0,53(1,90)$ & $22(81)$ \\
\hline Patul .............. & - & $\longrightarrow$ & 一 & 0,61 & 27 \\
\hline Golden Renet ....... & 一 & - & - & $0,94(1,90)$ & $40(81)$ \\
\hline Reinette Baumann .... & 一 & 一 & - & $0,77(1,17)$ & $33(50)$ \\
\hline Reinette de Champagne & - & - & 一 & $0,82(2,30)$ & $35(49)$ \\
\hline White Rosmarin ...... & 一 & $\ldots$ & - & $0,60(1,3)$ & $25(20)$ \\
\hline Signe Tillesch ....... & - & 一 & - & 1,12 & 48 \\
\hline Mespilus germanica..... & A $n / p / m$ & $\mathrm{IV}-\mathrm{V}$ & rose & 0,5 & 10 \\
\hline Morus alba $\ldots \ldots \ldots$ & A $p / n$ & V-VI & verdâtre & - & - \\
\hline Persica vulgaris........ & A $n / p / m$ & III-IV & rose pâle & $1,8-2,26$ & $4(3-8)$ \\
\hline
\end{tabular}




\begin{tabular}{|c|c|c|c|c|c|}
\hline Espèces et variétés & $\begin{array}{l}\text { Caractéristique } \\
\text { de la plante }\left(^{*}\right)\end{array}$ & $\begin{array}{c}\text { Période } \\
\text { de floraison } \\
\text { (mois) }\end{array}$ & $\begin{array}{l}\text { Couleur } \\
\text { des fleurs }\end{array}$ & $\begin{array}{l}\text { Sucre } \\
\text { par fleur } \\
(\mathrm{mg})\end{array}$ & $\begin{array}{c}\text { Miel } \\
(\mathrm{kg} / \mathrm{ha})\end{array}$ \\
\hline 1 & 2 & 3 & 4 & 5 & 6 \\
\hline Prunus domestica ....... & $\mathrm{A} n / p / m$ & $\mathrm{IV}-\mathrm{V}$ & blanc & $0,6-1,1$ & $20 \quad(24-40)$ \\
\hline Ana Späth ......... & - & - & - & $1,59(0,74)$ & $19(24)$ \\
\hline d'Agen $\ldots \ldots \ldots \ldots$ & - & 一 & 一 & $0,61(1,52)$ & t20(41) \\
\hline Bistritene . . . . . . . & - & - & 一 & 0,57 & 19 \\
\hline Bühe $\ldots \ldots \ldots \ldots$ & - & - & 一 & 0,48 & 16 \\
\hline Grasa romineasca ..... & 一 & - & $\ldots$ & $0,30(0,94)$ & $10(31)$ \\
\hline Mirabelle........... & - & 一 & - & $0,04(0,64)$ & $5(21)$ \\
\hline Reine Claude ........ & - & 一 & - & $0,25(0,75)$ & $8(25)$ \\
\hline Tuleu gras . . . . . . . . & $\longrightarrow$ & 一 & 一 & $0,3(0,8)$ & $10(6,0)$ \\
\hline Vinete rominesti ...... & - & - & - & $0,30(0,94)$ & $10(6,0)$ \\
\hline Vinat de Italia ...... & - & - & - & 0,64 & 21 \\
\hline Pyrus communis....... & A $n / p / m / c$ & III-V & - & 0,3 & $10(8-16)$ \\
\hline Bistritene $\ldots \ldots \ldots \ldots$ & - & 一 & 一 & 0,18 & 6 \\
\hline Bon Louis . . . . . . . . & 一 & 一 & — & $0,20(0,7)$ & $6(23)$ \\
\hline 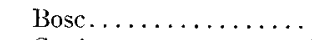 & 一 & - & - & $0,63(0,80)$ & $21(26)$ \\
\hline Curé............. & 一 & - & - & 1,14 & 46 \\
\hline Diel ............. & 一 & - & - & 0,92 & 30 \\
\hline Duchesse d'Angoulème. & - & 一 & - & $0,59(1,22)$ & $19(40)$ \\
\hline Favorita lui Clapp .... & A $n / p / m / c$ & III-V & white & $0,87(1,0)$ & $29(33)$ \\
\hline Hardempont ........ & - & - & - & 1,10 & 45 \\
\hline Japonez ........... & 一 & - & - & $0,17(1,0)$ & $6(33)$ \\
\hline Williams ........... & - & - & - & $0,16(0,94)$ & $5(31)$ \\
\hline Ribes sp. ............ & $\mathrm{A} n / p$ & IV $-V$ & verdâtre & 1,3 & 30 \\
\hline Cassis $\ldots \ldots \ldots \ldots \ldots$ & - & - & $\ldots$ & $1,1^{\prime} \mathrm{k}$ & 68 \\
\hline groseilles..$\ldots \ldots \ldots$ & 一 & - & 一 & 0,10 & 6,5 \\
\hline groseille blanche... & - & - & 一 & 0,10 & 6,5 \\
\hline Rubus caesius ........ & - & V-IX & blanc & 1,9 & $20(50)$ \\
\hline Rubus idaeus..... & A $n / p / m$ & VI-VII & - & 7,6 & $70(150)$ \\
\hline Sorbus sp. ........... & A $n / p / m$ & IV-V & - & 0,4 & $30-40$ \\
\hline Vitis vinifera $\ldots \ldots \ldots$ & A $n / p$ & V-VIII & verdâtre blanc & 一 & 5 \\
\hline
\end{tabular}

(*) Signes conventionnels: $\mathrm{A}=$ arbre

$a=$ arbuste

$n=$ nectar

$p=$ pollen

$m=$ miellat

$c=$ propolis

$\mathrm{V}-\mathrm{X}=$ mai-octobre

() Moyenne de production dans les années favorables.

Dans le tableau 2, nous exposons la valeur mellifère de I3 variétés de Pommiers. On constate que, sous le rapport de la production du nectar, il existe des différences remarquables entre les variétés étudiées. Ces différences mettent clairement en évidence la possibilité de définir des objectifs de sélection convergente en arboriculture fruitière. Ces objectifs sont importants, non seulement pour les espèces et les variétés autostériles, mais aussi pour celles qui sont autofertiles. Pour illustrer cette affirmation, nous exposons le cas du Cassis dont toutes les variétés sont autofertiles. Les recherches effectuées à 1'Institut de Miciürinsk ont montré qu'il existe des différences notables dans l'intensité des visites des Abeilles selon que la production de nectar est plus ou moins importante; on constate que les variétés de Cassis les plus recherchées par les Abeilles fournissent une grande quantité d'un nectar très 
E. V. SANDULEAC

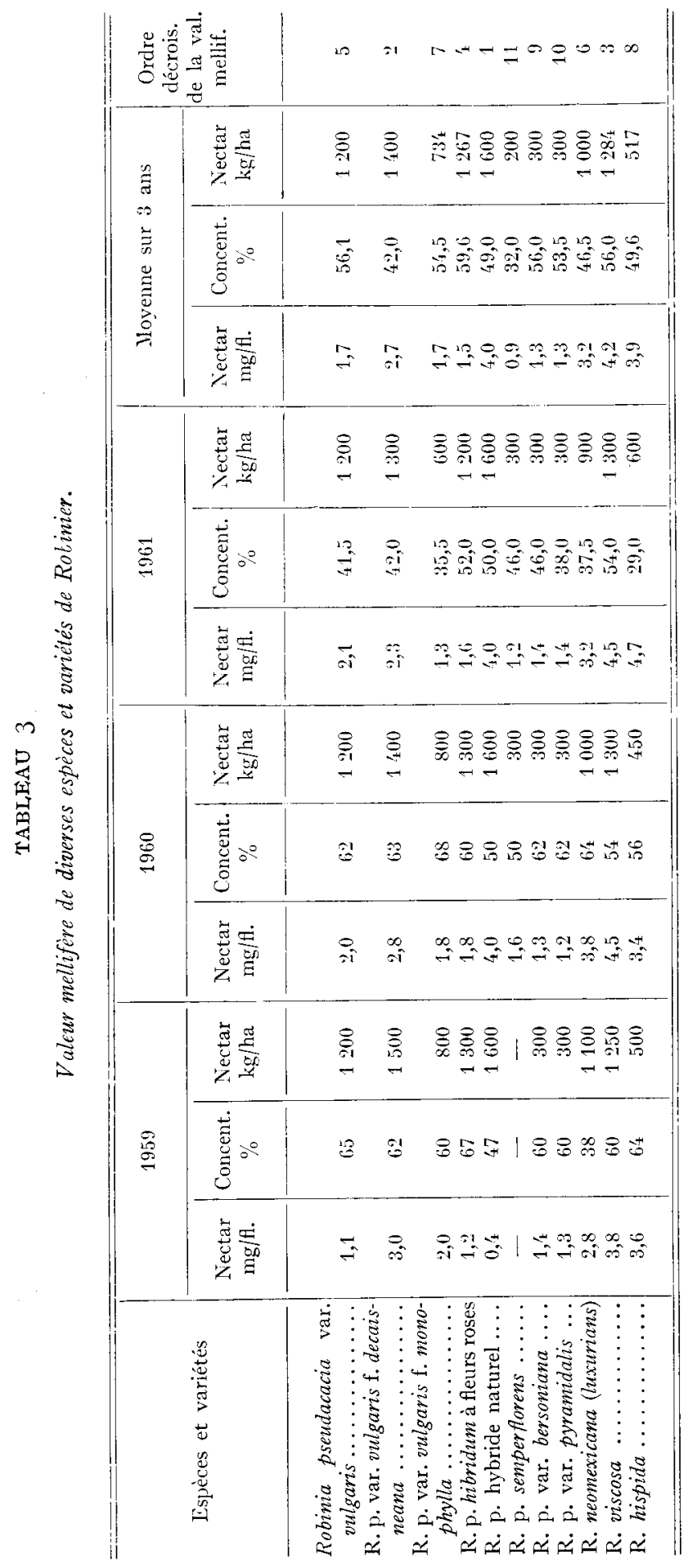


sucré. L'augmentation de $\mathrm{I} 6,7-20$ p. Ioo à $23,3-25,7$ p. Ioo de sucre dans le nectar a déterminé un accroissement du nombre des visites des Abeilles.

En conclusion, on peut affirmer que l'arboricultuture fruitière a le plus grand intérêt à utiliser les méthodes de la sélection convergente pour l'étude de la biologie florale des arbres fruitiers.

Parmi les espèces forestières, c'est le Robinier (Robinia) qui semble se prêter le mieux à la sélection convergente. Bien que le Robinier présente une grande importance apicole, la détermination de sa valeur mellifère n'a été faite que dernièrement. Les Abeilles visitent activement presque toutes les espèces du genre Robinier, à l'exception de la forme décorative (R. hispida) qui, malgré sa grande production de nectar, n'est recherchée que lorsque les plantes mellifères en fleurs manquent dans l'aire utile de butinage des Abeilles.

Les études entreprises à Bucarest sur les espèces et variétés de Robinier ont montré de grandes différences en ce qui concerne leur attractivité pour les Abeilles. Dans le tableau 3 on a groupé les données se rapportant à la valeur mellifère des différents Robiniers. Il en ressort que $R$. decaisneana est le plus productif, n'étant dépassé que par un hybride naturel de $R$. pseudoacacia et $R$. neomexicana. Les deux espèces $R$. neomexicana et $R$. viscosa ont montré une valeur mellifère remarquable. L'importance de cas deux espèces est d'autant plus grande qu'elles fleurissent plus tard que le Robinier commun. Sous le rapport de 1'attractivité pour les Abeilles, la première place revient à $R$. p. var-decaisneana suivi de $R$. neomexicana et de $R$. viscosa.

Les formes hybrides entre $R$. pseudoacacia, $R$. neomexicana et $R$.p. decaisneana sont très appréciées. Selon nos observations, la sélection convergente doit être orientée en fonction de la valeur mellifère du matériel initial en appliquant la sélection de

TABIEAU 4

Données biomitriques sur les fleurs de Robinier.

\begin{tabular}{|c|c|c|c|c|c|c|}
\hline Espèces & $\begin{array}{l}\text { Détermi- } \\
\text { nation (i) }\end{array}$ & $\begin{array}{c}\text { Nombre } \\
\text { de mesures }\end{array}$ & Min. & Max. & Moyenne & $\begin{array}{l}\text { Coefficient } \\
\text { de variation \% }\end{array}$ \\
\hline \multirow{5}{*}{ Robinia decaisneana } & 1 & 35 & 97 & 200 & $155,5 \pm 3,9$ & $1 ! k, 8$ \\
\hline & 2 & 35 & 48 & 66 & $57,0 \pm 0,63$ & 6,5 \\
\hline & 3 & 35 & 11 & 37 & $25,5 \pm 0,9^{\prime}$ & $2-2,1$ \\
\hline & 't & 200 & 20 & 26 & $23,1 \pm 0,07$ & 1,1 \\
\hline & 5 & $\because 00$ & 17 & 22 & $19,0 \pm 0,06$ & 4,8 \\
\hline \multirow{5}{*}{$\begin{array}{l}\text { Robinia unifolia (mono- } \\
\text { phylla) }\end{array}$} & 1 & 50 & 88 & 181 & $124,8 \pm 2,32$ & 13,8 \\
\hline & 2 & 50 & 50 & 62 & 56,0 上 0,51 & 6,4 \\
\hline & 3 & 50 & 8 & 18 & $13,3=0,333$ & 17,5 \\
\hline & $t^{\prime}$ & 200 & 18 & $2: 3$ & $20,8 \pm 0,05$ & 3,8 \\
\hline & 5 & 200 & 15 & 20 & $17,6 \div 0,08$ & 6,3 \\
\hline \multirow{5}{*}{ Robinia viscosa } & 1 & 35 & 71 & 120 & $104,5 \pm 2,05$ & 11,7 \\
\hline & $y$ & 35 & 37 & 35 & $47,0 \pm 0,85$ & 10,3 \\
\hline & 3 & 35 & $\geq 6$ & $4: 4$ & $31,6 \pm 0,58$ & 10,9 \\
\hline & '́t & 200 & 20 & 92 & $21,4 \pm 0,05$ & $3, t^{\prime}$ \\
\hline & 5 & 200 & 17 & 90 & $18,5 \pm 0,05$ & ${ }^{\prime},{ }^{\prime}+1$ \\
\hline
\end{tabular}

(1) $1=$ longueur de l'inflorescence (mm).

$2=$ diamètre de l'inflorescence $(\mathrm{mm})$.

$3=$ nombre de fleurs par inflorescence.

$\iota_{t}=$ longueur des fleurs (mm).

$5=$ largeur du vexilum (mm). 
masse aux sujets à floraison abondante ayant une forte production de nectar, ceci en vue d'une hybridation ayant pour effet d'augmenter la production de nectar et une floraison progressive qui prolonge la durée de la miellée.

Les objectifs de la sélection convergente ainsi établis s'identifient à ceux de la sélection du Robinier pour son extension à de nouvelles zones de culture.

Les hybrides qui proviennent du croisement de $R$. pseudacacia avec $R$. neomexicana ont une meilleure croissance que les parents et ils paraissent pleins d'avenir. Les faits exposés plus haut confirment la possibilité d'obtenir des formes hybrides mellifères précieuses ; de telles formes existent d'ailleurs déjà à l'heure actuelle.

\section{CONCLUSION}

Nous basant sur les recherches entreprises sur le Tournesol, le Robinier et le Pommier, nous arrivons aux conclusions suivantes quant aux possiblités d'application de la sélection convergente.

a) La sélection convergente constitue un procédé efficace tant en ce qui concerne l'augmentation de la production du nectar que sous le rapport de l'amélioration d'autres qualités intéressantes du point de vue purement agricole.

b) Les données contenues dans les tableaux I, 2 et 3 montrent l'existence d'une variabilité très large de la production nectarifère des espèces étudiées, ce qui permet d'appliquer la sélection convergente.

c) Chez le 'Tournesol, la corrélation entre le pourcentage d'huile et la production de nectar est positive, ce qui présente une grande importance pour l'application de la sélection convergente en vue de la production d'huile. Les sélectionneurs trouvent ainsi des possibilités supplémentaires d'observation de variétés nouvelles intéressantes

d) La sélection convergente doit être considérée comme une méthode complexe permettant de perfectionner l'adaptation fleur-Abeille par une action simultanée portant à la fois sur la plante (mise en évidence et fixation génétique des caractères conditionnant l'attractivité pour les Insectes) et sur l'Abeille (augmentation des capacités dé pollinisation). Réduire la sélection convergente à une simple amélioration d'ordre morphologique (allongement de la langue chez l'Abeille, raccourcissement du tube floral chez la plante) signifierait, pratiquement, la rendre non efficiente et applicable seulement au Trèfle violet.

e) La sélection convergente, à notre avis, signifie une sélection complexe de l'Abeille en vute d'accroître ses facultés de recherche des plantes cultivées entomophiles et une sélection de ces mêmes plantes en vue de l'augmentation maximum de leur valeur attractive à l'égard des Insectes pollinisateurs.

f) La voie la plus sûre pour la réalisation des objectifs de la sélection convergente est la sélection des plantes d'après leur production de nectar et leur multiplication en culture.

Dans le domaine de l'action à poursuivre pour l'augmentation de la production du nectar et de la culture des plantes agricoles entomophiles, nous pensons que le rôle déterminant doit revenir à la physiologie qui se doit d'approfondir notre connaissance du mécanisme intime de la sélection nectarifère. L'agrotechnique, de son côté, 
doit rechercher les procédés culturaux susceptibles d'intensifier la production de nectar des fleurs. Quant à la sélection, son objectif est de créer des variétés à grande capacité nectarifère et de promouvoir la culture des espèces qui sont les plus précieuses pour l'apiculture.

\title{
Rę̧u pour publication en janvier 1962.
}

\author{
SUMMARY \\ CONVERGENT SFLECTION, A PRACTICAL METHOD FOR INCREASING \\ THE EFFICIENCY OF POLIINISATION BY INSECTS
}

By convergent selection is meant the simultaneous action both on plants, with a view to heightening their attractiveness to the pollinating cntomofauna, and on the honey bee for intensifying its fuality as a pollinator.

The variability of nectar production in various species proves the possibility of creating new and highly melliferous species. Thus, for instance, the nectar production in sunflower was : local population : 23,940 kg honey per ha ; Vniimk 89.3 : 28,980; Vniimk 6540:35, 100; Armavir $3497: 62,640$. As to the melliferous value of trees, it is shown in tables 2 and $2 a$, and that of acacia in tables 3 and 4 wherefrom it follows that the highly melliferous species are : Robinia psetudacacia var. vulgaris $F$. decaisneana producing $\mathrm{I} 400 \mathrm{~kg} / \mathrm{ha}$ honey and being exceeded only by its hybrids, a fact proving once more the great possibilities this method of convergent selection has as to the continous perfecting of the unity "Pollinating insect-Blossom ".

\section{RÉFÉRENCES BIBLIOGRAPHIQUES}

AlbeNski A. V., 1959. Selection and production of seeds in forest species, Moscou.

Darwis Ch., i95.. The Origin of the Species, Publishing House og the Academy, Bucarest.

KOPELKIYLVSKI G. V., I96I. Increase of Nectar yield of entomophile plants by means of selection, $M e z d u n a-$ rodnyi Kongress po pcelozodsinu, 18, Moscou.

Lobacoy G. A., r $96 \mathrm{r}$. Creating new varieties of fruits-trees, Mloscou.

Procopowicz P. I., ig6o. Selected beekeeping works, Moscou.

Sanduleac I., ig6r. Achievenents and prospects in the selection of honey plants, Apicultura, 8, r2-16. 